\title{
Research on the Mechanism of Kaempferol for Treating Senile Osteoporosis by Network Pharmacology and Molecular Docking
}

\author{
Fuyu Tang, ${ }^{1,2}$ Peng Zhang $\mathbb{D}^{1,3}$ Wenhua Zhao, ${ }^{1,3}$ Guangye Zhu, ${ }^{1}$ Gengyang Shen, ${ }^{4}$ \\ Honglin Chen, ${ }^{1,3,4}$ Xiang Yu, ${ }^{4}$ Zhida Zhang, ${ }^{4}$ Qi Shang, ${ }^{1,3}$ De Liang, ${ }^{4}$ Xiaobing Jiang $\mathbb{D},{ }^{3,4}$ \\ and Hui Ren $\mathbb{D i D}^{3,4}$ \\ ${ }^{1}$ Guangzhou University of Chinese Medicine, Guangzhou 510405, China \\ ${ }^{2}$ Liuzhou Hospital of Chinese Medicine (Liuzhou Hospital of Zhuang Medicine), Guangxi Zhuang Autonomous Region, \\ Liuzhou, 545000, China \\ ${ }^{3}$ Lingnan Medical Research Center of Guangzhou University of Chinese Medicine, Guangzhou 510405, China \\ ${ }^{4}$ The First Affiliated Hospital of Guangzhou University of Chinese Medicine, Guangzhou 510405, China
}

Correspondence should be addressed to Xiaobing Jiang; spinedrjxb@sina.com and Hui Ren; renhuispine@163.com

Received 9 December 2021; Accepted 8 January 2022; Published 3 February 2022

Academic Editor: Xiang Liu

Copyright (c) 2022 Fuyu Tang et al. This is an open access article distributed under the Creative Commons Attribution License, which permits unrestricted use, distribution, and reproduction in any medium, provided the original work is properly cited.

Kaempferol (KP), as a natural anti-inflammatory compound, has been reported to have curative effects on alleviating senile osteoporosis (SOP), which is an inflammation-related musculoskeletal disease, but the molecular mechanisms remain unclear due to scanty relevant studies. We predicted the targets of KP and SOP, and the common targets of them were subsequently used to carry out PPI analysis. Moreover, we adopted GO and KEGG enrichment analysis and molecular docking to explore potential mechanisms of KP against SOP. There were totally $152 \mathrm{KP}$-related targets and 978 SOP-related targets, and their overlapped targets comprised 68 intersection targets. GO enrichment analysis showed 1529 biological processes $(p<0.05)$, which involved regulation of inflammatory response, oxidative stress, regulation of bone resorption and remodeling, osteoblast and osteoclast differentiation, etc. Moreover, KEGG analysis revealed 146 items including 44 signaling pathways $(p<0.05)$, which were closely linked to TNF, IL-17, NF-kappa B, PI3K-Akt, MAPK, estrogen, p53, prolactin, VEGF, and HIF-1 signaling pathways. By means of molecular docking, we found that kaempferol is bound with the key targets' active pockets through some connections such as hydrogen bond, pi-alkyl, pi-sigma, pi-pi Stacked, pi-pi T-shaped, and van der Waals, illustrating that kaempferol has close combination with the key targets. Collectively, various targets and pathways involve in the process of kaempferol treatment against SOP through regulating inflammatory response, oxidative stress, bone homeostasis, etc. Moreover, our study first reported that kaempferol may regulate core targets' expression with involvement of inflammatory response, oxidative stress, and bone homeostasis, thus treating SOP.

\section{Introduction}

Senile osteoporosis (SOP) is an inflammation-related musculoskeletal disease with serious complications including spine deformation, osteoporotic fracture, and bone pain $[1,2]$. Osteoporotic vertebral fracture (OVF) is the worstaffected complication in SOP patients with about 1.8 million vertebral fractures estimated happening every year in China, and the number of vertebral fractures is predicted to increase to 3 million in 2050 [3]. SOP poses serious threats to senior citizens' life and health, which adds to social and family burdens. The treatment of SOP involves the use of drugs inhibiting bone resumption clinically, but long-term use of these drugs can result in some complications, which limit their clinical application [4]. Recently, more and more scholars attach increasing attention to the osteoprotective effect of traditional Chinese medicine on treating SOP [5].

Kaempferol (KP, PubChem CID: 5280863) is a flavonoid identified in various natural products and traditional Chinese medicine like Drynariae Rhizoma [6]. KP has been 
reported to have the curative effect of treating SOP by acting on both osteoblasts and osteoclasts, which may exert osteogenic and antiosteoclastic effects [7]. The current study has illustrated that KP could influence adipogenesis [8], inflammation [9], oxidative stress [10], osteoblastic apoptosis [11], and osteoclastic apoptosis [12], resulting in osteoprotective effects. Therefore, KP could serve as a complementary and alternative medicine with a good prospect for clinical application on treating SOP.

In our prevent study, we performed bioinformatics analysis including network pharmacology and molecular docking so as to carry out systematic analysis on numerous pathways and targets involved in the function of $\mathrm{KP}$ on treating SOP.

\section{Materials and Methods}

Figure 1 describes the flow chart of study design.

2.1. Obtaining KP-Related Structure and Targets. We obtained the KP-related structure and targets through the following steps: first, we conducted data retrieval on the TCMSP database (https://tcmsp-e.com/) [13], which provides comprehensive information of $\mathrm{KP}$ including its structure and target information; second, by searching the PubChem database (https://pubchem.ncbi.nlm.nih.gov/), the KP structure was stored as an "SDF" file, which was imported into the SwissTargetPrediction database (http:// new.swisstargetprediction.ch/) [14] to get the targets associated with KP; and third, we adopted the UniProt database (http://www.uniprot.org/uniprot/) to standardize the KPrelated target proteins with "popular organisms" limited to humans, which were described as gene symbols.

2.2. SOP-Related Genes and Corresponding Proteins. The key word "Senile Osteoporosis" was searched in the two databases, including GeneCards (https://www.genecards.org/) [15] and Online Mendelian Inheritance in Man (OMIM, https://omim.org/) [16], with the species set as "Homo sapiens." The UniProt database was adopted to standardize the corresponding proteins of SOP-related genes.

2.3. Overlapped Target Proteins (OTPs). R (v3.6.1) software was used to take the overlap of KP- and SOP-related target proteins to get OTPs.

2.4. Protein Interaction Analysis of OTPs. The STRING database (https://string-db.org/) [17] was retrieved to get the protein-protein interaction (PPI) data of OTPs. Next, the PPI information of OTPs was input into Cytoscape (v3.7.2) software (https://www.cytoscape.org/) [18] to construct the PPI network and calculate the degrees of targets in the network through network topology analysis. We determined the target proteins with degree above average to be core target proteins. Afterwards, we generated a KP-OTPs-SOP network via Cytoscape.
2.5. GO Enrichment Analysis and KEGG Pathway Analysis. We conducted GO and KEGG analysis of the overlapped targets by means of clusterProfiler package (R3.6.1) and extracted the enrichment results with $p<0.05$.

2.6. Molecular Docking between Key Targets and KP. The top 5 proteins in terms of degree were chosen for molecular docking, which were considered the key targets in the process of KP treating SOP. In order to explore interaction activity between KP and its key targets, we utilized AutoDock Vina (v1.1.2) software [19] to carry out molecular docking simulations. We searched the PubChem database (https:// pubchem.ncbi.nlm.nih.gov/) for the 3D structure of KP. We used AutoDock Tools (v1.5.6) to distribute charge and combine nonpolar hydrogen for KP and converted the results into a PDBQT file. We downloaded the crystal structures of target proteins from the RCSB PDB website (https://www.rcsb. org/). Then, the target protein was separated from its ligand, added polar hydrogen, and distributed charge via AutoDock Tools, which would be subsequently stored as a PDBQT file. AutoDock Tools were also utilized to calculate the center and size of the docking box. Molecular docking simulations among KP and the target proteins were performed with every affinity calculated. Afterwards, Discovery Studio (https://www.3ds.com/products-services/biovia/products/ molecular-modeling-simulation/biovia-discovery-studio/) was used to draw and analyze the docking results of KP.

\section{Results}

3.1. KP-Related Structure and Target Proteins. From TCMSP and SwissTargetPrediction databases, we got 152 targets of KP. With them imported into the UniProt database, we obtained KP-related target proteins called gene symbols. Supplementary Tables S1 and S2 show the KP-related structure and target information.

3.2. Target Information of SOP and Overlapped Target Proteins (OTPs). Through the retrieval of GeneCards and OMIM databases, we obtained a total of 978 target proteins of SOP. We took the overlap of KP- and SOP-related targets as OTPs, which included 68 overlapped targets, as demonstrated in Table 1 and Figure 2(a).

3.3. PPI Network Construction and Core Target Protein Screening. OTPs were imported into the STRING database with the targets having no interactive connections with others hidden. And then we imported the PPI data into Cytoscape (v 3.7.2) to draw PPI network in Figure 2(b). There were 28 target proteins predicted to be the core target proteins (Table 2), whose degrees were above average degree (20.59).

3.4. KP-OTPs-SOP Network Plotting. Figure 2(c) shows the KP-OTPs-SOP network with 70 nodes and 136 edges included. In Figure 2(c), the red circular nodes stand for the overlapped target proteins (OTPs). The orange diamond node stands for "kaempferol." The yellow round rectangle 


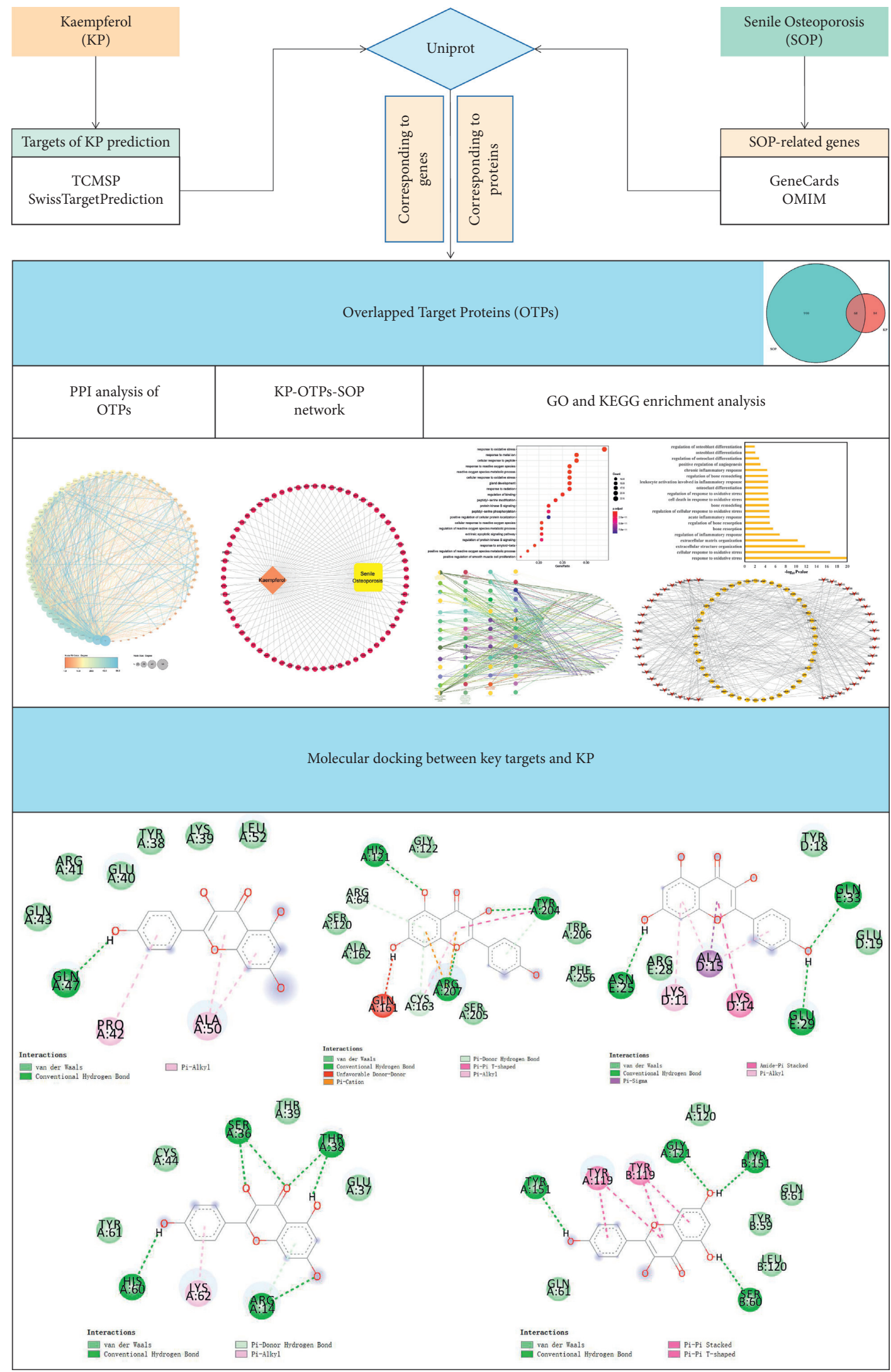

Figure 1: The flowchart of this study. 
TABLE 1: Potential target genes of KP in the treatment of SOP.

\begin{tabular}{|c|c|}
\hline Number & Gene \\
\hline 1 & NOS2 \\
\hline 2 & PTGS1 \\
\hline 3 & $\mathrm{AR}$ \\
\hline 4 & PPARG \\
\hline 5 & PTGS2 \\
\hline 6 & HSP90AA1 \\
\hline 7 & PIK3CG \\
\hline 8 & PRKACA \\
\hline 9 & DPP4 \\
\hline 10 & PGR \\
\hline 11 & F2 \\
\hline 12 & NOS3 \\
\hline 13 & RELA \\
\hline 14 & AKT1 \\
\hline 15 & BCL2 \\
\hline 16 & BAX \\
\hline 17 & TNF \\
\hline 18 & JUN \\
\hline 19 & CASP3 \\
\hline 20 & МАРК8 \\
\hline 21 & $\mathrm{XDH}$ \\
\hline 22 & MMP1 \\
\hline 23 & STAT1 \\
\hline 24 & HMOX1 \\
\hline 25 & CYP3A4 \\
\hline 26 & CYP1A1 \\
\hline 27 & ICAM1 \\
\hline 28 & VCAM1 \\
\hline 29 & ALOX5 \\
\hline 30 & AHR \\
\hline 31 & INSR \\
\hline 32 & GSTM1 \\
\hline 33 & SLPI \\
\hline 34 & NOX4 \\
\hline 35 & AKR1B1 \\
\hline 36 & TYR \\
\hline 37 & CA2 \\
\hline 38 & $\mathrm{ABCB} 1$ \\
\hline 39 & GLO1 \\
\hline 40 & SYK \\
\hline 41 & GSK3B \\
\hline 42 & MMP9 \\
\hline 43 & MMP2 \\
\hline 44 & CDK5 \\
\hline 45 & CCNB1 \\
\hline 46 & ESR2 \\
\hline 47 & TTR \\
\hline 48 & CYP19A1 \\
\hline 49 & EGFR \\
\hline 50 & IGF1R \\
\hline 51 & MPO \\
\hline 52 & PIK3R1 \\
\hline 53 & CA1 \\
\hline 54 & SRC \\
\hline 55 & PTK2 \\
\hline 56 & KDR \\
\hline 57 & MMP13 \\
\hline 58 & MMP3 \\
\hline 59 & MET \\
\hline 60 & BACE1 \\
\hline
\end{tabular}

TABle 1: Continued.

\begin{tabular}{lc}
\hline Number & Gene \\
\hline 61 & AKR1A1 \\
62 & APP \\
63 & PARP1 \\
64 & MMP12 \\
65 & ESR1 \\
66 & CFTR \\
67 & TERT \\
68 & MAPT \\
\hline
\end{tabular}

node stands for "senile osteoporosis." The edges stand for the interactive relationships among kaempferol, senile osteoporosis, and the overlapped targets.

3.5. GO Enrichment Analysis. We got 1529 items of biological process (BP). The top 20 items are shown in Figure 3(a). Noteworthily, we have filtrated 20 entries mainly related to inflammatory response, oxidative stress, angiogenesis, bone remodeling and resorption, and osteoblast and osteoclast differentiation, which have a close association with bone homeostasis as demonstrated in Figure 3(b). Additionally, we input 68 OTPs into Cytoscape for GO.BP enrichment analysis with $p$ value set to 0.00001 . Figure 3(c) illustrates the enrichment results mainly involved in the following four aspects: (i) inflammation-associated activities, such as regulation of reactive oxygen species metabolic process, reactive oxygen species biosynthetic process, and cellular response to oxidative stress; (ii) cell cycle, such as negative regulation of apoptotic signaling pathway and negative regulation of extrinsic apoptotic signaling pathway; (iii) angiogenesis, such as regulation of blood vessel endothelial cell migration; and (iv) physiological process, such as female gonad development and mammary gland development.

3.6. KEGG Pathway Analysis. The KEGG enrichment analysis of 68 target genes was performed using $\mathrm{R}$ software. We finally got a total of 146 items including the 44 key signaling pathways listed in Table 3 . We conducted network visualization via Cytoscape as plotted in Figure 3(d).

3.7. Molecular Docking Analysis. Among 28 core targets, the top 5 target proteins in terms of degree were chosen for molecular docking, including AKT1, TNF, SRC, CASP3, and JUN, which were considered the key targets in the process of $\mathrm{KP}$ treating SOP. To verify how KP binds to the key targets, we adopted molecular docking using AutoDock Vina to predict their docking interactions. Table 4 shows the docking results including affinity and interaction information.

According to Figure 4(a), KP combined with AKT1 by forming one hydrogen bond with the residue Gln-47 and six van der Waals interactions with Gln-43, Arg-41, Glu-40, Tyr-38, Lys-39, and Leu-52 (binding affinity: $-6.0 \mathrm{kcal} / \mathrm{mol}$ ). In addition, there were pi-alkyl interactions upon $\mathrm{KP}$ with Pro-42 and Ala-50. 

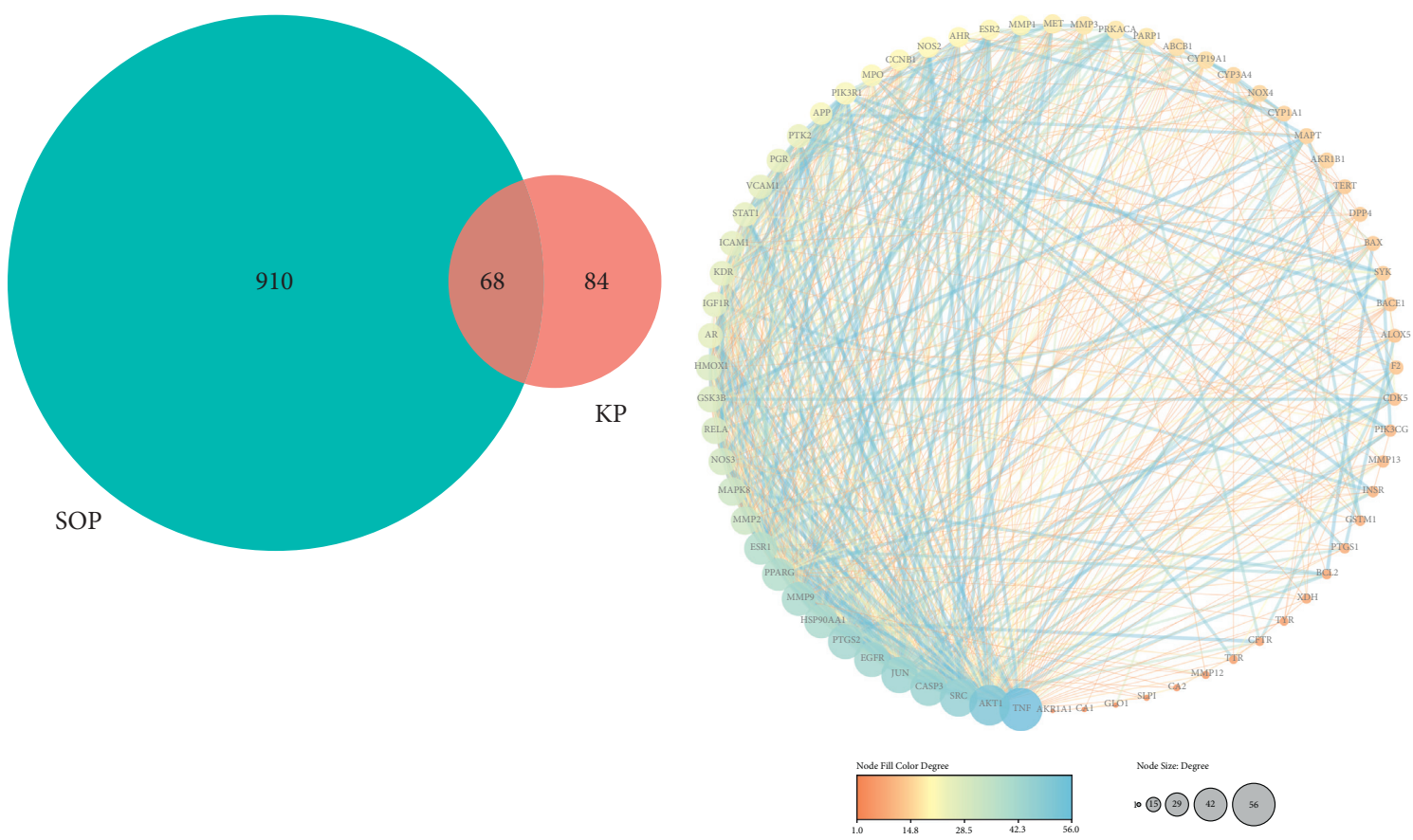

(a)

(b)

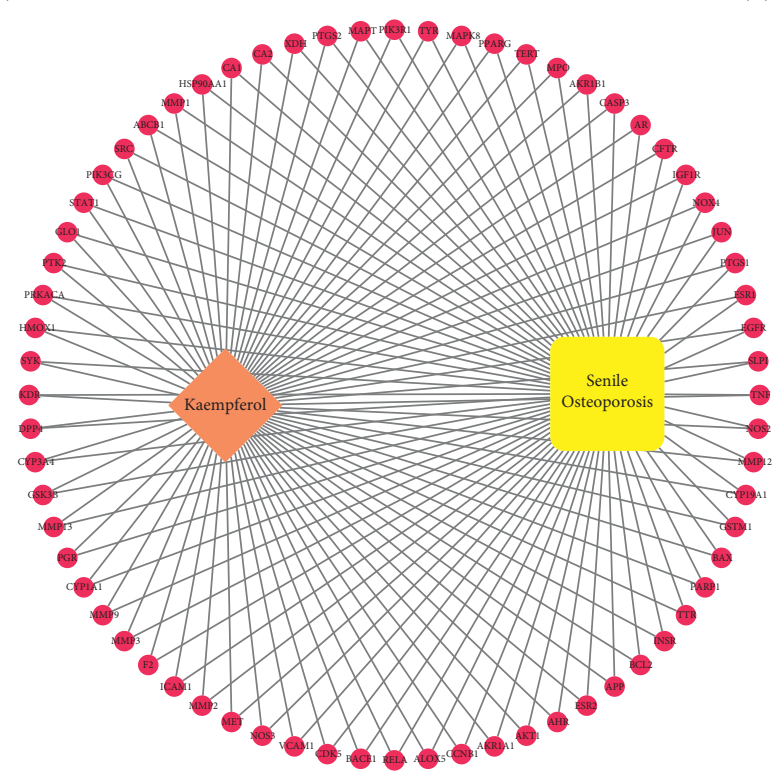

(c)

Figure 2: Venn diagram of OTPs (a), PPI network of OTPs (b), and KP-OTPs-SOP network (c).

According to Figure 4(b), the combination affinity of KP on TNF was $-7.6 \mathrm{kcal} / \mathrm{mol}$. The residues containing Leu- 120 , Gln-61, and Tyr-59 interacted with KP by forming 5 van der Waals interactions. Moreover, KP combined with TNF by forming four hydrogen bonds with the residues Gly-121, Ser-60, and Tyr-151. Notably, there were pi-pi stacked and pi-pi T-shaped interactions between KP and Tyr-119.

According to Figure 4(c), the combination affinity of $\mathrm{KP}$ on SRC was $-5.9 \mathrm{kcal} / \mathrm{mol}$. There existed pi-alkyl interaction and pi-donor hydrogen bond, respectively, provided by the Lys- 62 and Arg- 14 residues in the interactions with KP. Moreover, KP was bound with the residues Ser-36,
Thr-38, Arg-14, and His-60 by hydrogen bonds and Glu-37, Thr-39, Cys-44, and Tyr-61 by van der Waals.

According to Figure 4(d), the combination affinity of KP on CASP 3 was $-8.4 \mathrm{kcal} / \mathrm{mol}$. There were 6 van der Waals interactions provided by the Gly-122, Ser-120, Ala-162, Ser205, Phe-256, and Trp-206 residues in the interactions with KP. There existed pi-alkyl interaction and pi-donor hydrogen bonds provided by the Cys-163, Tyr-204, and Arg-64 residues in the interactions with KP. Additionally, KP combined with CASP3 by forming three hydrogen bonds with the residues Arg-207, His-121, and Tyr-204 and an unfavorable donor-donor interaction with Gln-161. 
TABLE 2: Core targets of KP in the treatment of SOP.

\begin{tabular}{lcc}
\hline Number & Core targets & Degree \\
\hline 1 & TNF & 56 \\
2 & AKT1 & 52 \\
3 & SRC & 47 \\
4 & CASP3 & 45 \\
5 & JUN & 45 \\
6 & EGFR & 43 \\
7 & PTGS2 & 43 \\
8 & HSP90AA1 & 41 \\
9 & MMP9 & 41 \\
10 & ESR1 & 39 \\
11 & PPARG & 39 \\
12 & MAPK8 & 32 \\
13 & MMP2 & 32 \\
14 & NOS3 & 30 \\
15 & RELA & 29 \\
16 & HMOX1 & 28 \\
17 & GSK3B & 28 \\
18 & KDR & 26 \\
19 & AR & 26 \\
20 & ICAM1 & 26 \\
21 & IGF1R & 26 \\
22 & STAT1 & 25 \\
23 & VCAM1 & 25 \\
24 & PGR & 24 \\
25 & PTK2 & 24 \\
27 & APP & 22 \\
28 & PIK3R1 & 22 \\
& MPO & 21 \\
\hline & &
\end{tabular}

Notably, there were pi-cation and pi-pi T-shaped interactions upon KP with Arg-207 and Tyr-204.

According to Figure 4(e), the combination affinity of KP on JUN was $-6.4 \mathrm{kcal} / \mathrm{mol}$. There existed 3 hydrogen bonds provided by the Asn-25, Glu-29, and Gln-33 residues in the interactions with KP. Additionally, there were 3 van der Waals interactions upon KP with Arg-28, Tyr-18, and Glu19. Notably, KP interacted with the Lys-11, Lys-14, and Ala15 residues by pi-sigma, pi-alkyl, and amide-pi stacked interactions.

\section{Discussion}

KP, a flavonoid identified in Drynariae Rhizoma, has been revealed to have beneficial effects on SOP via inhibiting osteoclast formation and bone loss [12, 20]. Studies have illustrated that KP exerts the antiosteoporotic function via upregulating microRNA-101 and activating the Wnt/ $\beta$-catenin pathway, which promotes osteoblast differentiation, proliferation, and migration [21]. To further explore the mechanisms of KP in treating SOP, we carried out a series of bioinformatics analysis to screen potential targets and pathways in the present study.

In our present study, we got 68 overlapped targets between KP and SOP, including 28 core targets listed in Table 2. According to PPI network topology analysis, we noticed that these targets were characteristics of inflammation, oxidative stress, and bone homeostasis-associated proteins. The top five targets ranked by degree are AKT1,
TNF, SRC, CASP3, and JUN, which are all bound tightly with $\mathrm{KP}$ according to molecular docking results, indicating that they may play a key role in KP treatment for SOP.

AKT1 (RAC-alpha serine/threonine-protein kinase) is identified as a unique signaling intermediate in bone homeostasis that controls the differentiation of osteoblasts and osteoclasts [22]. Some studies have verified that the inhibition of AKT1 expression would enhance bone turnover markers' expression and extracellular matrix mineralization, which consequently suppresses osteoporosis [23]. Moreover, AKT1 plays an important role in the PI3K-Akt signaling pathway, the involvement of which alleviates SOP progression by suppressing inflammatory response and osteoclast formation [24]. Moreover, evidence shows that kaempferol could block AKT1 phosphorylation [25]. Therefore, we speculated that KP could reduce inflammatory response and osteoclast formation by downregulating AKT1 expression levels in patients suffering from SOP, thus exerting therapeutic effects on SOP.

TNF (tumor necrosis factor) is the earliest inflammatory mediator produced in response to oxidative stress and promotes the production of inflammatory mediators and induces the expression of macrophage colony-stimulating factor (M-CSF) [26]. TNF affects SOP healing by activating NF-KB, promoting RANKL-induced osteoclast differentiation, and increasing bone resorption [27]. TNF- $\alpha$ plays a critical role in the development of osteoporosis via regulating oxidative stress, bone homeostasis, and remodeling $[28,29]$. Moreover, the existing study reveals that KP could significantly decrease the TNF expression and secretion [30]. Therefore, we speculated that KP could reduce oxidative stress in inflammatory response by downregulating TNF expression in SOP patients, so as to anti-SOP.

SRC (Proto-oncogene tyrosine-protein kinase Src) has been reported to involve in the process of osteoblast differentiation, which plays a vital role in advancing bone maturation [31]. Further studies have revealed that SRC plays a pivotal role in driving osteoblast proliferation and extracellular matrix (ECM) remodeling, which influences bone formation and remodeling [32]. Moreover, SRC is also an osteoclast-specific gene, which is essential for osteoclast function [33]. In general, the involvement of SRC exerts important effects on bone metabolism, which participates in the regulation of osteoblast and osteoclast activities [34]. Notably, sufficient evidence has revealed that KP regulates anti-inflammatory responses by the direct suppression of SRC [35]. However, research is needful to explore whether KP could exert therapeutic effects on SOP by regulating the expression of SRC and thus suppressing inflammatory response.

CASP3 (caspase-3) gets involved in cell apoptosis [36]. Evidence has revealed that the downregulation of CASP3 mRNA can promote SOP healing [37]. Further studies have demonstrated that the upregulation of CASP 3 can activate the p53 signaling pathway, destroy osteoblast maturation, and inhibit chondrocyte differentiation, thus restraining SOP healing [38]. It has been verified that CASP3 deletion could alleviate inflammatory response [39]. Moreover, it has been reported that KP treatment could remarkably decrease 


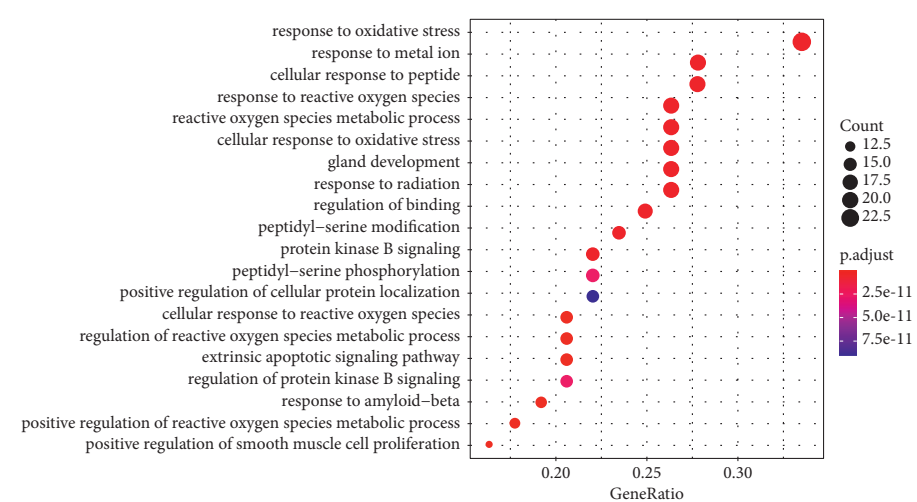

(a)

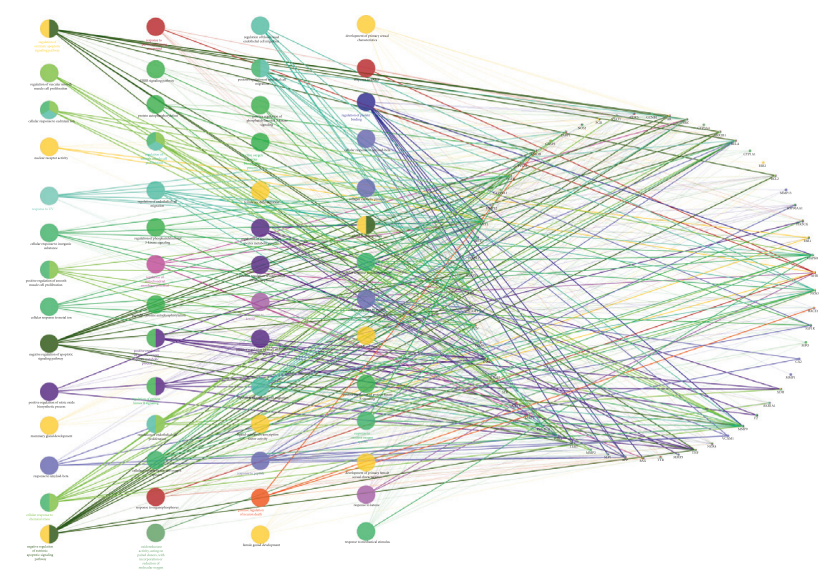

(c)

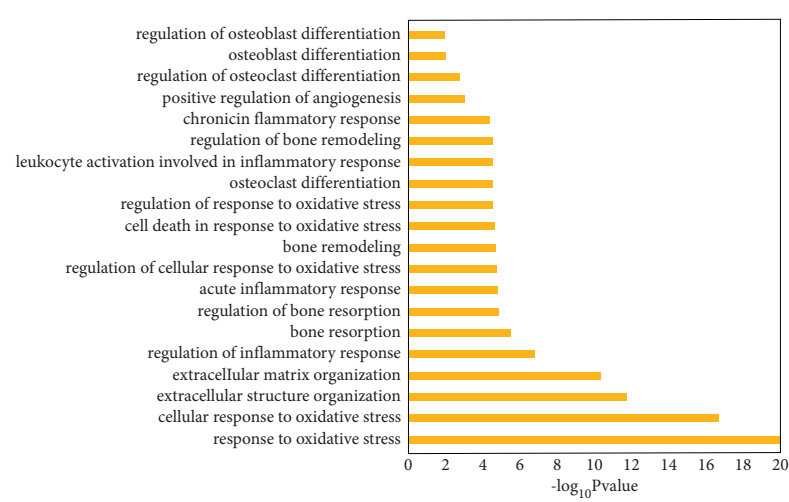

(b)

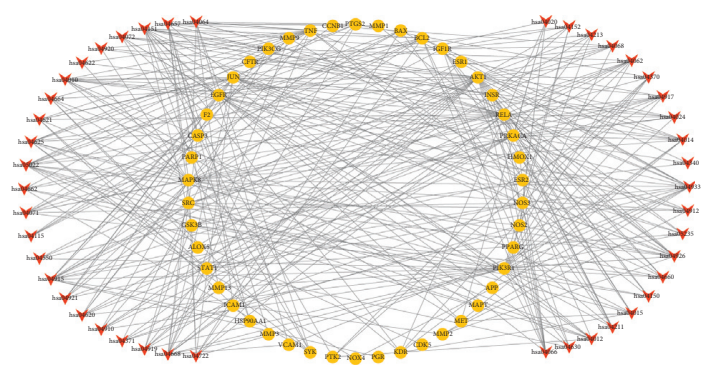

(d)

FIgURe 3: GO.BP enrichment analysis (a-c) and pathway-target network (d). (a, b) The top and screened 20 items of biological processes in terms of $p$ value. (c) Different colors represent different biological process groups, and node size stands for term $p$ value, while the edges represent the connections between biological processes and targets. (d) A red V-shaped node represents a signaling pathway, a yellow circular node represents a gene, and an edge represents a relationship between a pathway and a gene.

the CASP3 expression in vitro [40]. Nevertheless, there are scanty research projects exploring the regulation of KP on CASP3 expression to alleviate inflammatory response for treating SOP.

JUN is a proinflammatory factor and forms a dimer complex called AP-1 along with FOS, which accelerates the transcription and expression of genes related to bone growth and development containing AP-1 binding sites through multiple mechanisms, thus regulating bone metabolism $[41,42]$. Numerous studies have confirmed that the activation of JUN promotes osteoclastogenesis [43]. Moreover, JUN, as a regulatory factor in the JNK signaling pathway, could activate inflammatory response and osteoclast formation [44]. Notably, sufficient evidence has revealed that KP regulates anti-inflammatory responses by the suppression of JUN $[45,46]$. Thus, JUN plays a key role in inflammatory response and osteoclastogenesis and KP might treat SOP by suppressing JUN expression.

Similar to PPI analysis, GO enrichment results show consistent results. Additionally, biological processes involving the regulation of inflammatory response, oxidative stress, and bone homeostasis make a key role in KP treating SOP, as demonstrated in Figure 3(b). In recent years, reports have revealed that inflammatory response plays an important role in the pathogenesis of SOP, which could disrupt bone homeostasis by accelerating bone resorption and inhibiting bone formation, thereby triggering SOP [47]. Mounting evidence reveals the role of KP in attenuating inflammatory response by encumbering the expressions of inflammatory mediators in many signaling pathways like MAPK [48]. For example, the MAPK signaling pathway being activated would promote the expressions of inflammatory cytokines TNF- $\alpha$ and IL- $1 \beta$ in inflammatory response, while the presence of KP could suppress this pathway and exert protective effects on SOP [7, 49]. KP, modulating the activities of proinflammatory enzyme, has been reported to inhibit cyclooxygenase expression in numerous inflammatory disorders [50]. KP also suppresses the production of nitric oxide that triggers the activation of TNF- $\alpha$, thereby inhibiting inflammatory response [51]. There is growing evidence for the role of kaempferol in attenuating inflammatory response mediated by NF- $\mathrm{KB}$, indicating its protective effects on bone loss in postmenopausal osteoporosis by blocking TNF- $\alpha$-induced nuclear translocation of the NF-KB subunit p65 from the cytoplasm to the nucleus [52]. And KP could suppress age-related NF$\mathrm{KB}$ activation by inhibition of NF-KB subunit $\mathrm{p} 65$ translocation so as to restrain inflammatory response [53]. Some 
TABLE 3: KEGG pathway enrichment analysis.

\begin{tabular}{|c|c|c|c|}
\hline ID & Signaling pathway & Enriched gene number & $p$ value \\
\hline hsa04933 & AGE-RAGE signaling pathway & 15 & $1.25 E-15$ \\
\hline hsa04926 & Relaxin signaling pathway & 14 & $1.24 E-12$ \\
\hline hsa04915 & Estrogen signaling pathway & 14 & $3.18 E-12$ \\
\hline hsa04657 & IL-17 signaling pathway & 12 & $8.40 E-12$ \\
\hline hsa04668 & TNF signaling pathway & 12 & $6.95 E-11$ \\
\hline hsa04917 & Prolactin signaling pathway & 9 & $3.91 E-09$ \\
\hline hsa04625 & C-type lectin receptor signaling pathway & 10 & $8.98 E-09$ \\
\hline hsa04066 & HIF-1 signaling pathway & 10 & $1.42 E-08$ \\
\hline hsa04151 & PI3K-Akt signaling pathway & 15 & $1.04 E-07$ \\
\hline hsa04012 & ErbB signaling pathway & 8 & $3.64 E-07$ \\
\hline hsa04370 & VEGF signaling pathway & 7 & $4.16 E-07$ \\
\hline hsa04010 & MAPK signaling pathway & 13 & $5.19 E-07$ \\
\hline hsa04211 & Longevity regulating pathway & 8 & $5.21 E-07$ \\
\hline hsa04064 & NF-kappa B signaling pathway & 8 & $1.73 E-06$ \\
\hline hsa04071 & Sphingolipid signaling pathway & 8 & $4.78 E-06$ \\
\hline hsa04722 & Neurotrophin signaling pathway & 8 & $4.78 E-06$ \\
\hline hsa04014 & Ras signaling pathway & 10 & $1.56 E-05$ \\
\hline hsa04664 & Fc epsilon RI signaling pathway & 6 & $1.70 E-05$ \\
\hline hsa04620 & Toll-like receptor signaling pathway & 7 & $1.94 E-05$ \\
\hline hsa04660 & $\mathrm{T}$ cell receptor signaling pathway & 7 & $1.94 E-05$ \\
\hline hsa04062 & Chemokine signaling pathway & 9 & $2.23 E-05$ \\
\hline hsa04662 & B cell receptor signaling pathway & 6 & $4.96 E-05$ \\
\hline hsa04919 & Thyroid hormone signaling pathway & 7 & $5.18 E-05$ \\
\hline hsa05235 & PD-L1 expression and PD-1 checkpoint pathway & 6 & $7.88 E-05$ \\
\hline hsa04068 & FoxO signaling pathway & 7 & $8.60 E-05$ \\
\hline hsa05022 & Pathways of neurodegeneration & 13 & $9.65 E-05$ \\
\hline hsa04912 & GnRH signaling pathway & 6 & 0.000100782 \\
\hline hsa04213 & Longevity regulating pathway & 5 & 0.000138239 \\
\hline hsa04072 & Phospholipase D signaling pathway & 7 & 0.000184632 \\
\hline hsa04921 & Oxytocin signaling pathway & 7 & 0.000236022 \\
\hline hsa04015 & Rap1 signaling pathway & 8 & 0.000278775 \\
\hline hsa04152 & AMPK signaling pathway & 6 & 0.000406963 \\
\hline hsa04621 & NOD-like receptor signaling pathway & 7 & 0.000693065 \\
\hline hsa04910 & Insulin signaling pathway & 6 & 0.000822176 \\
\hline hsa04150 & mTOR signaling pathway & 6 & 0.001558955 \\
\hline hsa04024 & cAMP signaling pathway & 7 & 0.002012705 \\
\hline hsa04920 & Adipocytokine signaling pathway & 4 & 0.002323 \\
\hline hsa04115 & p53 signaling pathway & 4 & 0.002854606 \\
\hline hsa04371 & Apelin signaling pathway & 5 & 0.005314536 \\
\hline hsa04630 & JAK-STAT signaling pathway & 5 & 0.010013151 \\
\hline hsa04340 & Hedgehog signaling pathway & 3 & 0.010486159 \\
\hline hsa04020 & Calcium signaling pathway & 6 & 0.01301751 \\
\hline hsa04622 & RIG-I-like receptor signaling pathway & 3 & 0.019110112 \\
\hline hsa04550 & Signaling pathways regulating pluripotency of stem cells & 4 & 0.028737678 \\
\hline
\end{tabular}

TABLE 4: Molecular interactions of key targets and KP.

\begin{tabular}{|c|c|c|c|c|}
\hline Compound & Target & PDB ID & $\begin{array}{c}\text { Affinity } \\
(\mathrm{kcal} / \mathrm{mol})\end{array}$ & Interactions \\
\hline Kaempferol & AKT1 & 1UNQ & -6.0 & Hydrogen bond, pi-alkyl, van der Waals \\
\hline Kaempferol & $\mathrm{TNF}$ & $2 \mathrm{AZ} 5$ & -7.6 & Hydrogen bond, van der Waals, pi-pi stacked, pi-pi T-shaped \\
\hline Kaempferol & SRC & 1041 & -5.9 & Hydrogen bond, pi-alkyl, van der Waals \\
\hline Kaempferol & CASP3 & 1NMS & -8.4 & $\begin{array}{c}\text { Hydrogen bond, pi-alkyl, pi-pi T-shaped, pi-cation, unfavorable donor-donor, van der } \\
\text { Waals }\end{array}$ \\
\hline Kaempferol & JUN & $5 \mathrm{FV} 8$ & -6.4 & Hydrogen bond, pi-alkyl, pi-sigma, van der Waals, amide-pi stacked \\
\hline
\end{tabular}

evidence showed that there was a negative correlation between dietary intake level of KP and serum CRP level, suggesting the key role of $\mathrm{KP}$ in reducing the risk of inflammation [54]. It has been verified that oxidative stress makes key functions in SOP-related inflammatory response [55]. Oxidative stress can alter bone homeostasis, accelerate bone resorption, and reduce bone formation, leading to the progression of SOP [56]. And some evidence has illustrated 


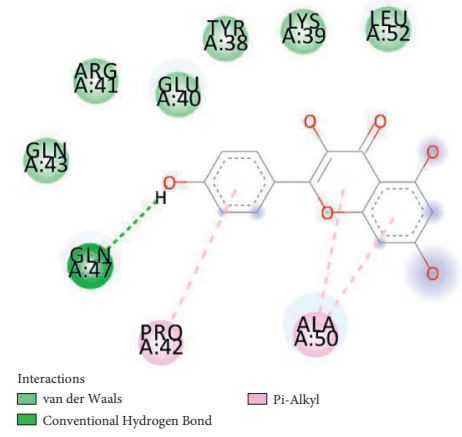

(a)

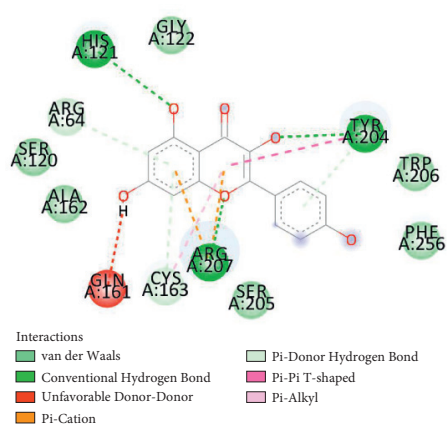

(d)

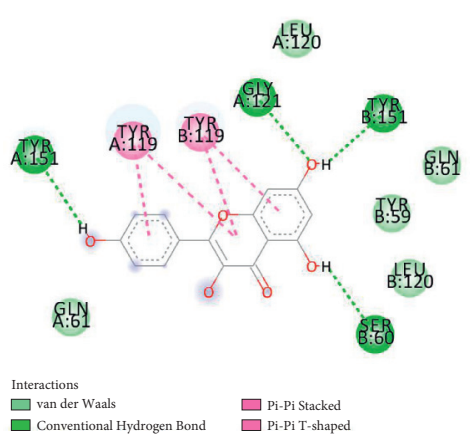

(b)

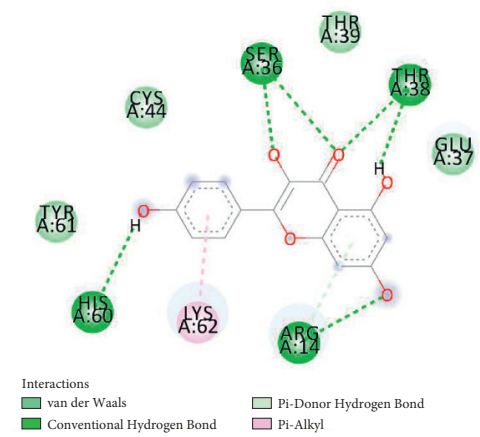

(c)

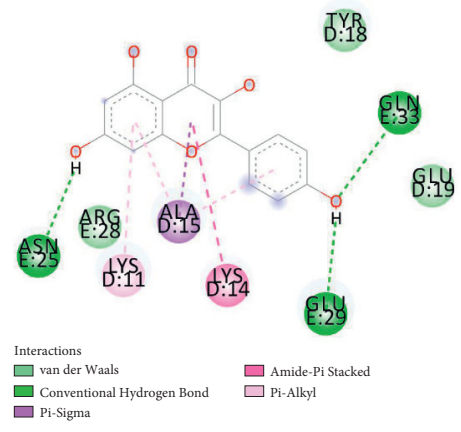

(e)

Figure 4: Simulated molecular docking of kaempferol on AKT1 (a), TNF (b), SRC (c), CASP3 (d), and JUN (e).

the therapeutic effects of kaempferol on the damage induced by oxidative stress and inflammation in osteoporosis [7, 57], suggesting that kaempferol is a natural antioxidant for treating osteoporosis. According to our present study, kaempferol may be an antioxidant with a good prospect that helps reduce inflammatory response and oxidative stress, thus improving SOP. Moreover, accumulating studies have revealed that the expressions of core targets, including AKT1 [58], TNF [29], SRC [35], CASP3 [59], JUN [44], etc., make vital functions in regulating inflammatory response and oxidative stress. Therefore, we speculated that KP could regulate core targets' expressions and bone homeostasis by inflammatory response and oxidative stress in SOP patients, so as to anti-SOP.

KEGG enrichment results revealed that PI3K-Akt, MAPK, VEGF, prolactin, HIF-1, TNF, estrogen, IL-17, p53, and NF-kappa B (NF-KB) signaling pathways may exert regulatory functions on kaempferol against SOP.

The involvement of PI3K-Akt signaling pathway alleviates SOP progression by suppressing inflammatory response and osteoclast formation [24]. Moreover, some studies have shown that the PI3K-Akt signaling pathway is involved in the inhibition of osteoporosis through promoting osteoblast proliferation, differentiation, and bone formation $[60,61]$, Therefore, the PI3K-AKT signaling pathway is essential in bone homeostasis.

Inflammatory pathways including IL-17 [62], TNF [63], and NF-KB [64] signaling pathways participate in regulating osteoclast differentiation. Moreover, the IL-17 signaling pathway can stimulate the synthesis of TNF- $\alpha$, IL-6, and NF$\mathrm{KB}$, thereby promoting RANKL-induced osteoclast differentiation [65]. Therefore, IL-17, TNF, and NF-KB signaling pathways are speculated to exert important functions in the process of KP treatment against SOP, which needs further identification.

The estrogen signaling pathway can exert regulatory functions on osteoblasts' and osteoclasts' proliferation, apoptosis, and differentiation [66]. The current study has revealed that KP regulates osteoblastic differentiation via estrogen receptor signaling [67]. Similar to the estrogen signaling pathway, research on the prolactin signaling pathway also indicates that KP has the function of estrogen regulation, which is evidence for kaempferol in treating postmenopausal SOP [68].

Evidence has confirmed that the activation of p53 signaling pathway can disrupt osteoblast maturation and restrain chondrocyte differentiation [38]. The inhibition of MAPK signaling pathway suppresses osteoclastogenesis [69]. The activation of HIF-1/VEGF signaling pathway can accelerate angiogenesis in bone tissues, which gets involved in the pathological evolution of SOP $[70,71]$.

In summary, our results predict some potential therapeutic targets and pathways, providing reference for future studies on KP treatment against SOP. However, one limitation of this study is that further in vivo and in vitro experiments are needed to confirm our findings.

\section{Conclusion}

Collectively, our results first reveal that KP may treat SOP possibly via regulating inflammatory response, oxidative stress, bone homeostasis, etc. These results will provide 
theoretical basis for KP treatment against SOP. However, the specific mechanism and material basis still need to be further verified in vivo and in vitro.

\section{Data Availability}

The data sets used and analyzed during the current study are available from the first author on reasonable request.

\section{Conflicts of Interest}

The authors declare that there are no conflicts of interest.

\section{Authors' Contributions}

All the authors listed participated in study conception, design, data analysis, and drafting the manuscript. All the authors read and approved the submitted manuscript. Fuyu Tang, Peng Zhang, and Wenhua Zhao contributed equally to this work.

\section{Acknowledgments}

This project was generously supported by the grants from the National Natural Science Foundation of China (81774338 and 81904225), Guangdong Natural Science Foundation Project (2021A1515011247), Guangxi Natural Science Foundation Project (2020GXNSFAA259082), Guangdong Province Universities and Colleges Pearl River Scholar Funded Scheme (GDUPS 2018), Medical Research Foundation of Guangdong Province (A2021320), Youth Scientific Research Training Project of GZUCM (2019QNPY04), Key Project of Basic Research and Applied Basic Research of Department of Education of Guangdong Province (2018KZDXM021), and Graduate Research Innovation Project of Guangzhou University of Chinese Medicine (A12606-21-429-001Z22).

\section{Supplementary Materials}

Supplementary Table S1: the structure of kaempferol. Supplementary Table S2: targets of kaempferol. (Supplementary Materials)

\section{References}

[1] Z. Z. Liu, C. G. Hong, W. B. Hu et al., “Autophagy receptor OPTN (optineurin) regulates mesenchymal stem cell fate and bone-fat balance during aging by clearing FABP3," Autophagy, vol. 17, no. 10, pp. 2766-2782, 2021.

[2] Y. Guo, X. Jia, Y. Cui et al., "Sirt3-mediated mitophagy regulates AGEs-induced BMSCs senescence and senile osteoporosis," Redox Biology, vol. 41, Article ID 101915, 2021.

[3] L. Si, T. M. Winzenberg, Q. Jiang, M. Chen, and A. J. Palmer, "Projection of osteoporosis-related fractures and costs in China: 2010-2050," Osteoporosis International, vol. 26, no. 7, pp. 1929-1937, 2015.

[4] E. F. Eriksen, J. Halse, and M. H. Moen, "New developments in the treatment of osteoporosis," Acta Obstetricia et Gynecologica Scandinavica, vol. 92, no. 6, pp. 620-636, 2013.
[5] X.-F. Tang, Z.-T. Ma, Y.-Y. Gao et al., "Systemic osteoprotective effects of Epimedii Folium and Ligustri Lucidi Fructus in senile osteoporosis rats by promoting the osteoblastogenesis and osteoclastogenesis based on MLP-ANN model," Chinese Medicine, vol. 15, no. 1, p. 87, 2020.

[6] Z. L. Xu, M. Y. Xu, H. T. Wang et al., "Pharmacokinetics of eight flavonoids in rats assayed by UPLC-MS/MS after oral administration of Drynariae rhizoma extract," Journal of Analytical Methods in Chemistry, vol. 2018, Article ID 4789196, 11 pages, 2018.

[7] S. K. Wong, K.-Y. Chin, and S. Ima-Nirwana, "The osteoprotective effects of kaempferol: the evidence from in vivo and in vitro studies," Drug Design, Development and Therapy, vol. 13, pp. 3497-3514, 2019.

[8] J. Zhu, H. Tang, Z. Zhang et al., "Kaempferol slows intervertebral disc degeneration by modifying LPS-induced osteogenesis/adipogenesis imbalance and inflammation response in BMSCs," International Immunopharmacology, vol. 43, pp. 236-242, 2017.

[9] W.-S. Lee, E.-G. Lee, M.-S. Sung, and W.-H. Yoo, "Kaempferol inhibits IL-1 $\beta$-stimulated, RANKL-mediated osteoclastogenesis via downregulation of MAPKs, c-fos, and NFATc1," Inflammation, vol. 37, no. 4, pp. 1221-1230, 2014.

[10] K. S. Suh, E.-M. Choi, M. Kwon et al., "Kaempferol attenuates 2-deoxy-d-ribose-induced oxidative cell damage in MC3T3E1 osteoblastic cells," Biological and Pharmaceutical Bulletin, vol. 32, no. 4, pp. 746-749, 2009.

[11] I.-R. Kim, S.-E. Kim, H.-S. Baek et al., "The role of kaempferolinduced autophagy on differentiation and mineralization of osteoblastic MC3T3-E1 cells," BMC Complementary and Alternative Medicine, vol. 16, no. 1, p. 333, 2016.

[12] C. J. Kim, S. H. Shin, B. J. Kim et al., "The effects of kaempferolinhibited autophagy on osteoclast formation," International Journal of Molecular Sciences, vol. 19, pp. 125-1, 2018.

[13] J. Ru, P. Li, J. Wang et al., "TCMSP: a database of systems pharmacology for drug discovery from herbal medicines," Journal of Cheminformatics, vol. 6, no. 1, p. 13, 2014.

[14] D. Gfeller, A. Grosdidier, M. Wirth, A. Daina, O. Michielin, and V. Zoete, "SwissTargetPrediction: a web server for target prediction of bioactive small molecules," Nucleic Acids Research, vol. 42, no. W1, pp. W32-W38, 2014.

[15] G. Stelzer, N. Rosen, I. Plaschkes et al., "The GeneCards suite: from gene data mining to disease genome sequence analyses," Current protocols in bioinformatics, vol. 54, pp. 1-33, 2016.

[16] J. S. Amberger and A. Hamosh, "Searching online mendelian inheritance in man (OMIM): a knowledgebase of human genes and genetic phenotypes," Current protocols in bioinformatics, vol. 58, pp. 1-12, 2017.

[17] C. v. Mering, M. Huynen, D. Jaeggi, S. Schmidt, P. Bork, and B. Snel, "STRING: a database of predicted functional associations between proteins," Nucleic Acids Research, vol. 31, no. 1, pp. 258-261, 2003.

[18] P. Shannon, A. Markiel, O. Ozier et al., "Cytoscape: a software environment for integrated models of biomolecular interaction networks," Genome Research, vol. 13, no. 11, pp. 2498-2504, 2003.

[19] O. Trott and A. J. Olson, "AutoDock Vina: improving the speed and accuracy of docking with a new scoring function, efficient optimization, and multithreading," Journal of Computational Chemistry, vol. 31, no. 2, pp. 455-461, 2010.

[20] B. Nowak, A. Matuszewska, A. Nikodem et al., "Oral administration of kaempferol inhibits bone loss in rat model of ovariectomy-induced osteopenia," Pharmacological Reports, vol. 69, no. 5, pp. 1113-1119, 2017. 
[21] Y. Wang, H. Chen, and H. Zhang, "Kaempferol promotes proliferation, migration and differentiation of MC3T3-E1 cells via up-regulation of microRNA-101," Artificial Cells, Nanomedicine, and Biotechnology, vol. 47, no. 1, pp. 10501056, 2019.

[22] A. Mukherjee and P. Rotwein, "Selective signaling by Akt1 controls osteoblast differentiation and osteoblast-mediated osteoclast development," Molecular and Cellular Biology, vol. 32, no. 2, pp. 490-500, 2020.

[23] P. Cai, Y. Lu, Z. Yin, X. Wang, X. Zhou, and Z. Li, "Baicalein ameliorates osteoporosis via AKT/FOXO1 signaling," Aging, vol. 13, no. 13, pp. 17370-17379, 2021.

[24] L. Zha, L. He, Y. Liang et al., "TNF- $\alpha$ contributes to postmenopausal osteoporosis by synergistically promoting RANKL-induced osteoclast formation," Biomedicine \& Pharmacotherapy, vol. 102, pp. 369-374, 2018.

[25] E. Jo, S. J. Park, Y. S. Choi, W.-K. Jeon, and B.-C. Kim, "Kaempferol suppresses transforming growth factor- $\beta 1$-induced epithelial-to-mesenchymal transition and migration of A549 lung cancer cells by inhibiting akt1-mediated phosphorylation of Smad3 at threonine-179," Neoplasia, vol. 17, no. 7, pp. 525-537, 2015.

[26] H. Kitaura, P. Zhou, H. J. Kim, D. V. Novack, F. P. Ross, and S. L. Teitelbaum, "M-CSF mediates TNF-induced inflammatory osteolysis," Journal of Clinical Investigation, vol. 115, no. 12, pp. 3418-3427, 2005.

[27] R. C. Schulman, A. J. Weiss, and J. I. Mechanick, "Nutrition, bone, and aging: an integrative physiology approach," Current Osteoporosis Reports, vol. 9, no. 4, pp. 184-195, 2011.

[28] B. A. Abdel-Wahab and M. E. Metwally, "Clozapine-induced cardiotoxicity: role of oxidative stress, tumour necrosis factor alpha and NF- $\kappa \beta$," Cardiovascular Toxicology, vol. 15, no. 4, pp. 355-365, 2015.

[29] C. Sang, Y. Zhang, F. Chen et al., "Tumor necrosis factor alpha suppresses osteogenic differentiation of MSCs by inhibiting semaphorin $3 \mathrm{~B}$ via $\mathrm{Wnt} / \beta$-catenin signaling in estrogen-deficiency induced osteoporosis," Bone, vol. 84, pp. 78-87, 2016.

[30] M. Palacz-Wrobel, P. Borkowska, M. Paul-Samojedny et al., "Effect of apigenin, kaempferol and resveratrol on the gene expression and protein secretion of tumor necrosis factor alpha (TNF- $\alpha$ ) and interleukin-10 (IL-10) in RAW-264.7 macrophages," Biomedicine \& Pharmacotherapy, vol. 93, pp. 1205-1212, 2017.

[31] G. V. O. Fernandes, A. D. M. Cavagis, C. V. Ferreira et al., "Osteoblast adhesion dynamics: a possible role for ROS and LMW-PTP," Journal of Cellular Biochemistry, vol. 115, no. 6, pp. 1063-1069, 2014.

[32] S. M. Barneze Costa, G. da Silva Feltran, V. Namba et al., "Infraphysiological $17 \beta$-estradiol (E2) concentration compromises osteoblast differentiation through Src stimulation of cell proliferation and ECM remodeling stimulus," Molecular and Cellular Endocrinology, vol. 518, Article ID 111027, 2020.

[33] X. He, L. Zhu, L. An, and J. Zhang, "MiR-143 inhibits osteoclastogenesis by targeting RANK and NF- $\kappa$ B and MAPK signaling pathways," Current Molecular Pharmacology, vol. 13, no. 3, pp. 224-232, 2020.

[34] J. H. Kim, K. Kim, I. Kim, S. Seong, and N. Kim, "c-Srcdependent and -independent functions of matk in osteoclasts and osteoblasts," The Journal of Immunology, vol. 200, no. 7, pp. 2455-2463, 2018.

[35] S. H. Kim, J. G. Park, J. Lee et al., "The dietary flavonoid Kaempferol mediates anti-inflammatory responses via the Src, Syk, IRAK1, and IRAK4 molecular targets," Mediators of Inflammation, vol. 2015, Article ID 904142, 15 pages, 2015.
[36] S. A. Lakhani, A. Masud, K. Kuida et al., "Caspases 3 and 7: key mediators of mitochondrial events of apoptosis," Science, vol. 311, no. 5762, pp. 847-851, 2006.

[37] W.-j. Liu, Z.-m. Jiang, Y. Chen et al., "Network pharmacology approach to elucidate possible action mechanisms of Sinomenii Caulis for treating osteoporosis," Journal of Ethnopharmacology, vol. 257, Article ID 112871, 2020.

[38] J. Wu, Y. Yang, Y. He et al., "EFTUD2 gene deficiency disrupts osteoblast maturation and inhibits chondrocyte differentiation via activation of the p53 signaling pathway," Human Genomics, vol. 13, no. 1, p. 63, 2019.

[39] Y. Li, Y. Yuan, Z.-X. Huang et al., "GSDME-mediated pyroptosis promotes inflammation and fibrosis in obstructive nephropathy," Cell Death \& Differentiation, vol. 28, no. 8, pp. 2333-2350, 2021.

[40] Y. Ling, H. Xu, N. Ren et al., "Prediction and verification of the major ingredients and molecular targets of tripterygii radix against rheumatoid arthritis," Frontiers in Pharmacology, vol. 12, Article ID 639382, 2021.

[41] N. Hannemann, J. Jordan, S. Paul et al., "The AP-1 transcription factor c-jun promotes arthritis by regulating cyclooxygenase- 2 and arginase-1 expression in macrophages," The Journal of Immunology, vol. 198, no. 9, pp. 3605-3614, 2017.

[42] M. A. Al Mamun, M. M. H. Asim, M. A. Z. Sahin, and M. A. A. Al-Bari, "Flavonoids compounds from Tridax procumbens inhibit osteoclast differentiation by down-regulating c-Fos activation," Journal of Cellular and Molecular Medicine, vol. 24, no. 4, pp. 2542-2551, 2020.

[43] Q. Zhang, X. Tang, Z. Liu et al., "Hesperetin prevents bone resorption by inhibiting RANKL-induced osteoclastogenesis and jnk mediated irf-3/c-jun activation," Frontiers in Pharmacology, vol. 9, Article ID 1028, 2018.

[44] K. M. Lee, C. Y. Lee, G. Zhang, A. Lyu, and K. K. M. Yue, "Methylglyoxal activates osteoclasts through JNK pathway leading to osteoporosis," Chemico-Biological Interactions, vol. 308, pp. 147-154, 2019.

[45] T. Behl, K. Mehta, A. Sehgal et al., "Exploring the role of polyphenols in rheumatoid arthritis," Critical Reviews in Food Science and Nutrition, pp. 1-22, 2021.

[46] C. Wall, R. Lim, M. Poljak, and M. Lappas, "Dietary flavonoids as therapeutics for preterm birth: luteolin and kaempferol suppress inflammation in human gestational tissues in vitro," Oxidative Medicine and Cellular Longevity, vol. 2013, Article ID 485201, 13 pages, 2013.

[47] N. Jiang, J. An, K. Yang et al., "NLRP3 inflammasome: a new target for prevention and control of osteoporosis?" Frontiers in Endocrinology, vol. 12, Article ID 752546, 2021.

[48] K. P. Devi, D. S. Malar, S. F. Nabavi et al., "Kaempferol and inflammation: from chemistry to medicine," Pharmacological Research, vol. 99, pp. 1-10, 2015.

[49] Z. Sun, Q. Li, R. Hou et al., "Kaempferol-3-O-glucorhamnoside inhibits inflammatory responses via MAPK and NF- $\kappa \mathrm{B}$ pathways in vitro and in vivo," Toxicology and Applied Pharmacology, vol. 364, pp. 22-28, 2019.

[50] J.-H. Lee and G.-H. Kim, "Evaluation of antioxidant and inhibitory activities for different subclasses flavonoids on enzymes for rheumatoid arthritis," Journal of Food Science, vol. 75, no. 7, pp. H212-H217, 2010.

[51] H. S. Rho, A. K. Ghimeray, D. S. Yoo et al., "Kaempferol and kaempferol rhamnosides with depigmenting and anti-inflammatory properties," Molecules, vol. 16, no. 4, pp. 3338-3344, 2011.

[52] P. Ramesh, R. Jagadeesan, S. Sekaran, A. Dhanasekaran, and S. Vimalraj, "Flavonoids: classification, function, and 
molecular mechanisms involved in bone remodelling," Frontiers in Endocrinology, vol. 12, Article ID 779638, 2021.

[53] Y. Qu, X. Li, F. Xu et al., "Kaempferol alleviates murine experimental colitis by restoring gut microbiota and inhibiting the LPS-TLR4-NF- $\kappa$ b Axis," Frontiers in Immunology, vol. 12, Article ID 679897, 2021.

[54] O. K. Chun, S.-J. Chung, K. J. Claycombe, and W. O. Song, "Serum C-reactive protein concentrations are inversely associated with dietary flavonoid intake in U.S. adults," Journal of Nutrition, vol. 138, no. 4, pp. 753-760, 2008.

[55] S. Zhu, W. Wei, Z. Liu, Y. Yang, and H. Jia, "Tanshinone-IIA attenuates the deleterious effects of oxidative stress in osteoporosis through the NF- $\kappa \mathrm{B}$ signaling pathway," Molecular Medicine Reports, vol. 17, no. 5, pp. 6969-6976, 2018.

[56] Y. Zhang, Y. Jiang, Y. Luo, and Y. Zeng, "Interference of miR212 and miR-384 promotes osteogenic differentiation via targeting RUNX2 in osteoporosis," Experimental and Molecular Pathology, vol. 113, Article ID 104366, 2020.

[57] H. Yao, J. Sun, J. Wei, X. Zhang, B. Chen, and Y. Lin, "Kaempferol protects blood vessels from damage induced by oxidative stress and inflammation in association with the Nrf2/HO-1 signaling pathway," Frontiers in Pharmacology, vol. 11, Article ID 1118, 2020.

[58] C. Lu, L. Liu, S. Chen et al., "Azathioprine pretreatment ameliorates myocardial ischaemia reperfusion injury in diabetic rats by reducing oxidative stress, apoptosis, and inflammation," Clinical and Experimental Pharmacology and Physiology, vol. 48, no. 12, pp. 1621-1632, 2021.

[59] Y. Akyuva, M. Nazıroğlu, and K. Yıldızhan, "Selenium prevents interferon-gamma induced activation of TRPM2 channel and inhibits inflammation, mitochondrial oxidative stress, and apoptosis in microglia," Metabolic Brain Disease, vol. 36, no. 2, pp. 285-298, 2021.

[60] J.-M. Pan, L.-G. Wu, J.-W. Cai, L.-T. Wu, and M. Liang, "Dexamethasone suppresses osteogenesis of osteoblast via the PI3K/Akt signaling pathway in vitro and in vivo," Journal of Receptors and Signal Transduction, vol. 39, no. 1, pp. 80-86, 2019.

[61] J.-C. Xi, H.-Y. Zang, L.-X. Guo et al., "The PI3K/AKT cell signaling pathway is involved in regulation of osteoporosis," Journal of Receptors and Signal Transduction, vol. 35, no. 6, pp. 640-645, 2015.

[62] D. Daoussis, A. P. Andonopoulos, and S.-N. C. Liossis, "Wnt pathway and IL-17: novel regulators of joint remodeling in rheumatic diseases. Looking beyond the RANK-RANKLOPG axis," Seminars in Arthritis and Rheumatism, vol. 39, no. 5, pp. 369-383, 2010.

[63] C.-h. Li, Z.-z. Ma, L.-l. Jian et al., "Iguratimod inhibits osteoclastogenesis by modulating the RANKL and TNF- $\alpha$ signaling pathways," International Immunopharmacology, vol. 90, Article ID 107219, 2021.

[64] S. Lin, X. 1. Zhao, and Z. Wang, "TANK-binding kinase 1 mediates osteoclast differentiation by regulating $\mathrm{NF}-\kappa \mathrm{B}$, MAPK and Akt signaling pathways," Immunology \& Cell Biology, vol. 99, no. 2, pp. 223-233, 2021.

[65] Y. Wang, J. Xu, X. Zhang et al., "TNF- $\alpha$-induced LRG1 promotes angiogenesis and mesenchymal stem cell migration in the subchondral bone during osteoarthritis," Cell Death \& Disease, vol. 8, no. 3, Article ID e2715, 2017.

[66] R. Y. Kim, H. J. Yang, Y. M. Song, I. S. Kim, and S. J. Hwang, "Estrogen modulates bone morphogenetic protein-induced sclerostin expression through the Wnt signaling pathway," Tissue Engineering. Part A, vol. 21, no. 13-14, pp. 2076-2088, 2015.
[67] A. J. Guo, R. C. Choi, K. Y. Zheng et al., "Kaempferol as a flavonoid induces osteoblastic differentiation via estrogen receptor signaling," Chinese Medicine, vol. 7, no. 1, p. 10, 2012.

[68] G. N. Hendy, H. Kaji, H. Sowa, J.-J. Lebrun, and L. Canaff, "Menin and TGF- $\beta$ superfamily member signaling via the smad pathway in pituitary, parathyroid and osteoblast," Hormone and Metabolic Research, vol. 37, no. 6, pp. 375-379, 2005.

[69] X. Li, Y. Wang, L. Li, S. Zhou, and F. Zhao, "Sclareol inhibits RANKL-induced osteoclastogenesis and promotes osteoblastogenesis through promoting $\mathrm{CCN} 1$ expression via repressing the MAPK pathway," Cell Biology and Toxicology, vol. 37, pp. 849-871, 2021.

[70] H. Zhong, C. Cao, J. Yang, and Q. Huang, "Research on relationship of HIF-1 signaling pathway and postmenstrual osteoporosis," Sichuan Da Xue Xue Bao Yi Xue Ban, vol. 48, no. 6, pp. 862-868, 2017.

[71] Y. Chen, B. Zhao, Y. Zhu, H. Zhao, and C. Ma, "HIF-1-VEGFnotch mediates angiogenesis in temporomandibular joint osteoarthritis," American Journal of Tourism Research, vol. 11, no. 5, pp. 2969-2982, 2019. 